\title{
Configuration and Performance of a Local Electrode Atom Probe
}

T. F. Kelly, T. T. Gribb, J. D. Olson, E. Oltman, S. A. Wiener, D. R. Lenz, J. D. Shepard, R. L. Martens, R. M. Ulfig, E. M. Strennen, J. H. Bunton, D. R. Strait, T. C. Kunicki, T. Payne, and J. Watson.

Imago Scientific Instruments Corporation, 6300 Enterprise Lane, Madison, WI 53719, imago.com

Two dedicated LEAP prototype instruments have recently been built and tested. Their design follows configurations proposed previously [1,2]. These instruments represent a significant departure from prior atom probe designs and so their key performance parameters are of interest.

These prototype LEAPs are ultrahigh vacuum (UHV) systems with a three stage vacuum system. This dry vacuum system uses titanium sublimation pumping with a large ion pump on the main chamber to achieve $3 \times 10^{-9} \mathrm{~Pa}$ after a bake at $150^{\circ} \mathrm{C}$. An intermediate chamber achieves $10^{-7} \mathrm{~Pa}$ with a magnetically-levitated turbopump. Eighteen specimens may be inserted at one time through the airlock and it takes less than ten minutes to complete insertion of a specimen into the main chamber. The specimen stage has $10 \mathrm{~nm}$ or smaller steps with $8 \mathrm{~mm}$ travel using an inchworm actuator on each of three axes. A vibration-isolated two stage helium refrigerator system cools the specimen to $27 \mathrm{~K}$ or lower.

The configuration of the optics is shown in Fig. 1. The extraction field is independent of the final acceleration field. A fixed tip-to-detector distance of $40 \mathrm{~mm}$ is used in the first prototype while a variable specimen-to-detector distance of 70 to $120 \mathrm{~mm}$ is used in the second prototype. The imaging detector uses a $40 \mathrm{~mm}$ diameter microchannel plate to achieve a large solid angle. With post acceleration of the ions, over $80^{\circ}$ full angle for detection is achieved as demonstrated in Fig. 2. A crossed delay line anode is used for ion detection. This anode achieves better than $800 \times 800$ pixel resolution with a data rate capability of over $10^{6}$ events per second.

Because the extraction voltage in the local electrode configuration is significantly lower than in conventional atom probes, the pulse voltage is proportionally lower and this makes it possible to build much higher repetition rate pulsers. Imago's pulser routinely operates at 1 x $10^{5}$ pulses per second. The electronics train needed to record data at this rate has also been developed. Sustained data collection rates of $1 \times 10^{6}$ atoms per minute with low errors are routinely utilized. An image recorded at this high data rate is shown in Fig. 3. This image of a model nickel-base alloy is approximately $87 \mathrm{~nm}$ diameter by $260 \mathrm{~nm}$ long and contains 55 million atoms [3]. There are over 300 precipitates of about $10 \mathrm{~nm}$ diameter in this image as shown by an isoconcentration contour for 10 atomic $\% \mathrm{Al}$.

A key element of the LEAP is the local extraction aperture. The size of the aperture opening influences the magnitude of the field enhancement and the ability to analyze microtips. To date, apertures as small as 20 micron diameter have been utilized to record images from both needles and microtips on planar surfaces.

Mass resolution of better than one part in $300 \mathrm{FWHM}$ has been demonstrated on the first prototype LEAP. Fig. 4 shows a mass spectrum that demonstrates one part in 346 FWHM at $\mathrm{W}_{186}^{+3}$ (mass-tocharge ratio=62). Mass resolution of one part in $500 \mathrm{FWHM}$ is expected in the second prototype LEAP at longer flight distances.

[1] T. F. Kelly et al., US patent \#5,440,124 (1995).

[2] T. F. Kelly et al., Ultramicroscopy 62 (1996) 29-42. 
[3] This specimen is from the work of D. Seidman of Northwestern University.

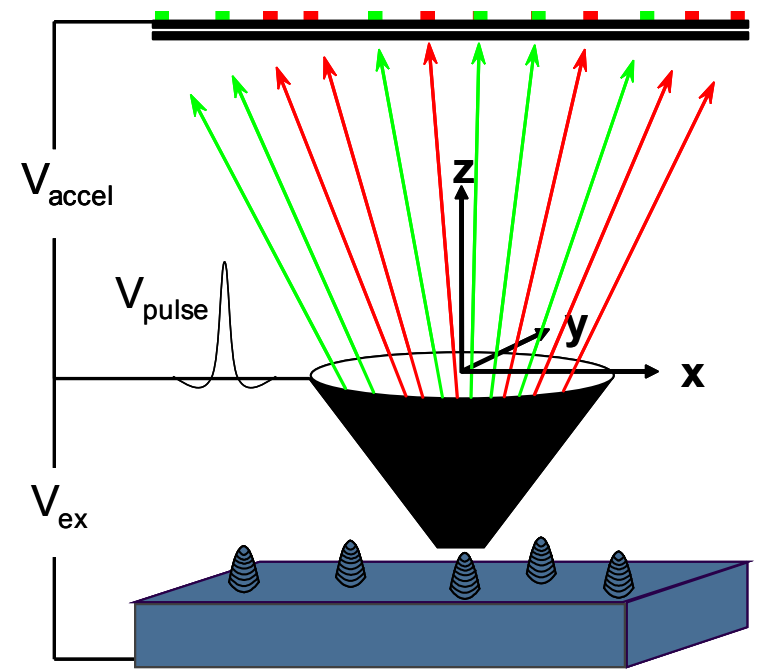

Fig. 1 Schematic of LEAP optics.

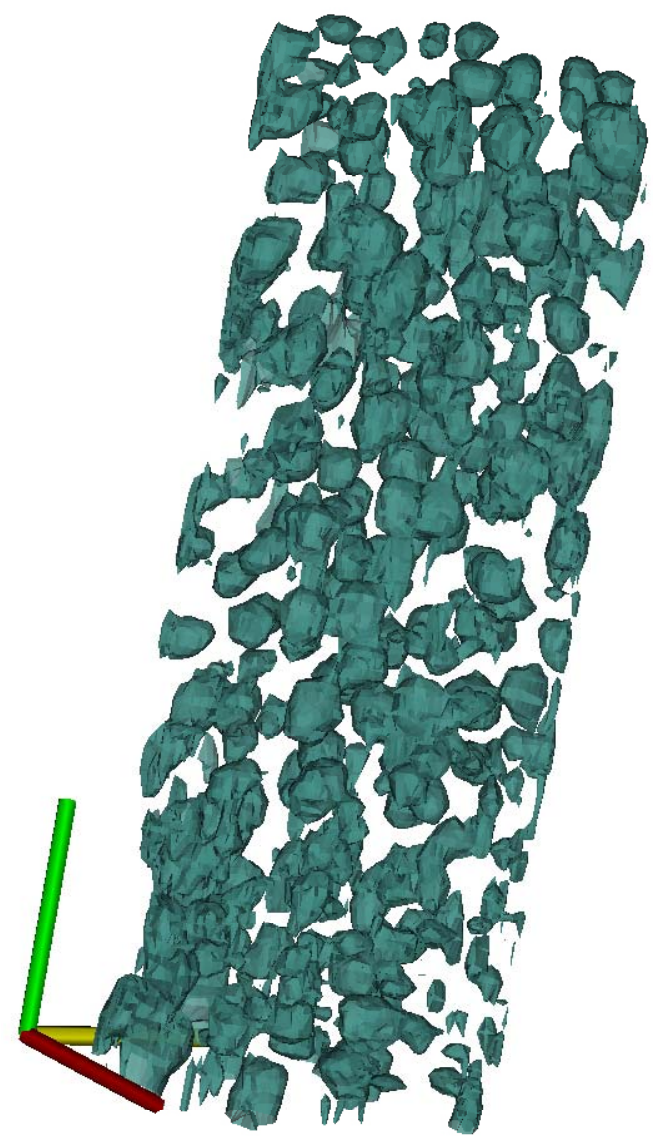

Fig. 3 LEAP 3D image of a nickel-base alloy with aluminum-rich precipitates shown as isoconcentration contours at 10 at $\% \mathrm{Al}$ [3].

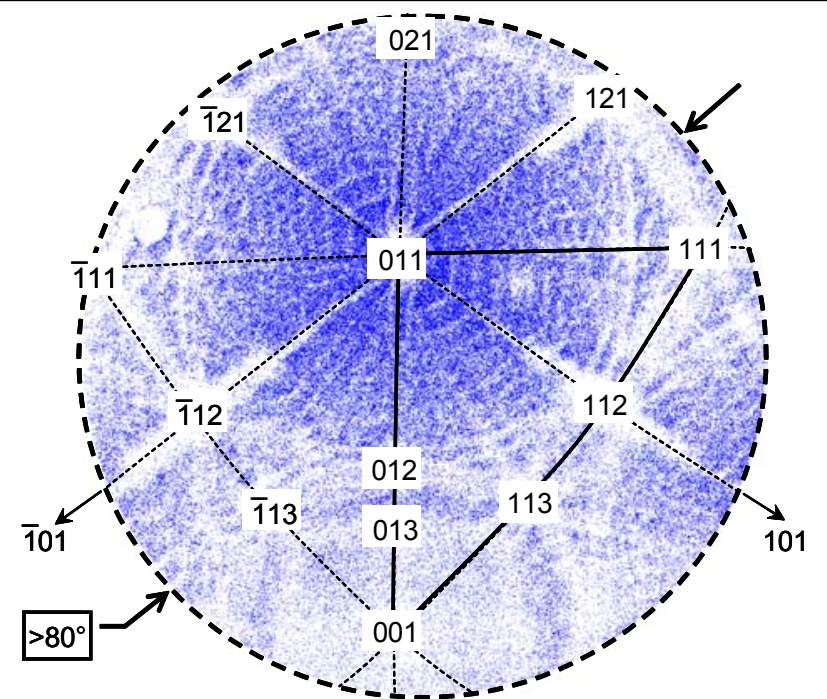

Fig. 2 Field evaporation image of tungsten with zone axes marked. This view is looking down the evaporation direction.

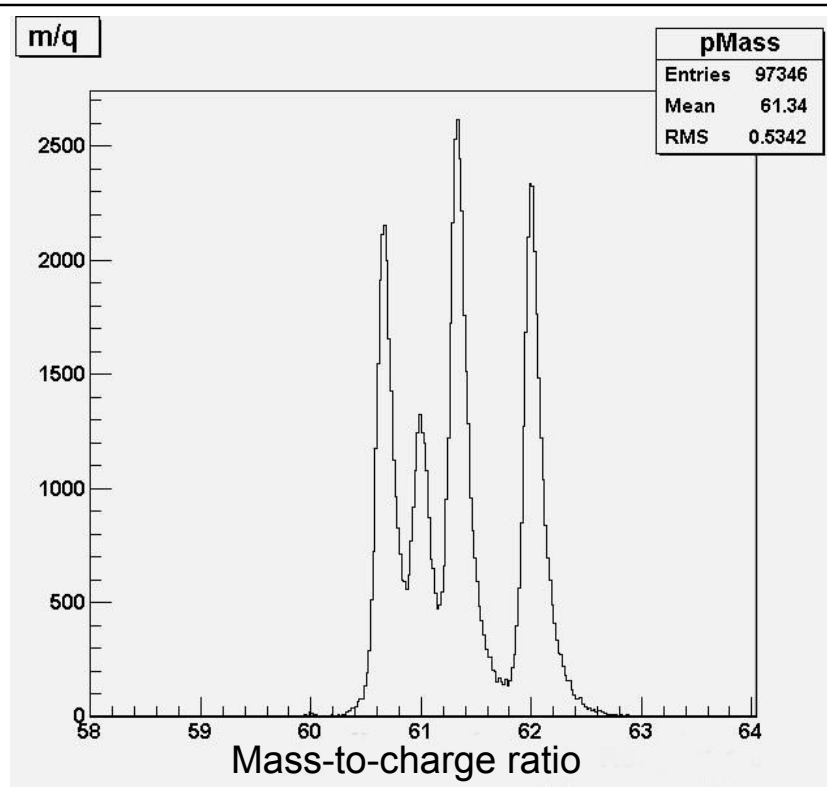

Fig. 4 A mass spectrum from a tungsten test specimen showing peaks of the triply ionized $\mathrm{W}$ (mass $=182,183,184$, and $186 \mathrm{amu}$ ). The FWHM mass resolution is measured as one part in 346 on the $\mathrm{W}_{186}{ }^{+3}$. 